Sharif University of Technology
Scientia Iranica
Transactions E: Industrial Engineering
hCIENTIA

\title{
An effective solution approach to multi-objective fractional fixed charge problem with fuzzy parameters
}

\author{
A. Mahmoodirad ${ }^{\mathrm{a}, *}$, S. Niroomand ${ }^{\mathrm{b}}$, and F. Hosseinzadeh Lotfic \\ a. Department of Mathematics, Masjed-Soleiman Branch, Islamic Azad University, Masjed-Soleiman, Iran. \\ b. Department of Industrial Engineering, Firouzabad Institute of Higher Education, Firouzabad, Fars, Iran. \\ c. Department of Mathematics, Science and Research Branch, Islamic Azad University, Tehran, Iran.
}

Received 9 January 2018; received in revised form 17 November 2018; accepted 31 December 2018

\author{
KEYWORDS \\ Fixed charge problem; \\ Fractional objective \\ function; \\ Fuzzy theory; \\ Goal; \\ programming.
}

\begin{abstract}
A multi-objective fixed charge problem in the presence of several fractional objective functions with triangular fuzzy parameters is considered in this study. The same problem has previously been tackled by Upmanyu and Saxena [1] adopting a method containing wrong mathematical concepts (see the commentary of Kaur and Kumar [2]). To overcome these shortcomings in the literature, an effective solution approach based on a typical goal programming approach is proposed to solve the problem for obtaining a Paretooptimal solution. The proposed approach considers the shortcomings of the method of Upmanyu and Saxena [1] and applies no ranking function of fuzzy numbers. In addition, the goal programming stage considers no preference of the decision maker. The computational experiments provided by an example from the literature prove effectiveness of the proposed approach.
\end{abstract}

(C) 2020 Sharif University of Technology. All rights reserved.

\section{Introduction}

The fixed charge problem was first introduced in [3]. It was a typical mixed-integer programming problem in which there were some fixed charge values for the variables that took positive amounts in a given feasible solution. As the fixed charges are used in the objective function of the fixed charge problem, the problem has a high degree of non-linearity that should be linearized using additional constraints. With regard to the wide range of applications of the fixed charge problem to business and engineering fields, the fixed charge may have different interpretations and measures. For

\footnotetext{
*. Corresponding author.

E-mail address: alimahmoodirad@gmail.com (A. Mahmoodirad)
}

doi: $10.24200 /$ sci. 2018.50229 .1588 example, in the field of facility planning problems, the fixed charge can be defined as establishing cost of a facility; in the field of transportation problems, it may be the fixed cost of sending an amount of product between two nodes; and in the field of scheduling problems, set-up times can be considered for the fixed charges.

As the fixed charge problem is an interesting problem in the field of optimization, it has been tackled by many researchers through different approaches. Steinberg [4] solved some small-size instances of the problem adopting a branch and bound algorithm. In some other studies like [5-7], the approximation based methods have been of interest in solving the fixed charge problem. The problem consists of a minimization concave objective function over a convex set of feasible solution areas. Therefore, in more recent studies, the fixed charge problem has been considered as a fixed charge transportation problem and solution 
approaches consisting of finding the extreme points and ranking their objective function values in order to find the global optimal solution have been considered for solving them (see [8]). Notably, in such solution approaches, in the case of non-degenerated problems, any extreme point would be a local optimal solution. Recently, fixed charge problems with both concepts of fixed and variable costs were used in the network flow problems by Gendron and Larose [9] and in the facility location problems by Fernández and Landete [10] (see also [11,12]). Furthermore, Arora [13] introduced a systematic approach that found an exact solution to the fixed charge problem. For more information on fixed charge based problems, the studies [14-16] can be referred to.

On the other hand, the fixed charge problem becomes more difficult when considering a fractional objective function. In linear programming, considering a fractional objective function turns it to a non-linear form (see $[17,18])$. In such cases, the problem becomes more difficult to solve exactly. As an example of fractional objective function, the objective function achieved by dividing unit based benefit by unit based investment can be mentioned, which is applicable to economical environments. Turning back to the fixed charge problems, Almogy and Levin [19] introduced a fixed charge problem with fractional objective function that might have many applications, e.g., to measuring any economic criterion by a fraction. The study considered the ship routing problem and maximized the profit gained in a unit of time in a route. For more details on the problems with fractional objective function, the studies [20,21] can be referred to.

This study focuses on a typical version of the fractional fixed charge problem. A fixed charge problem with several fractional objective functions in which each objective function includes triangular fuzzy parameters is to be solved. It is named Fuzzy Multi-objective Fractional Fixed Charge Problem (FMFFCP). FMFFCP has previously been tackled by Upmanyu and Saxena [1] using a fuzzy ranking based method, which contained wrong mathematical concepts. The shortcomings and errors of the method of [1] have been discussed in a commentary offered by Kaur and Kumar [2]. However, no serious study has been performed on FMFFCP. The main contribution of this study is solving FMFFCP by a goal programming approach, which uses no ranking function of fuzzy numbers. The approach adopts a linearization technique for each objective function, separately, to find its related goal. Then, a typical goal programming approach is employed for finding a Pareto-optimal solution to the FMFFCP. Finally, the performance of the proposed approach is evaluated by a numerical example taken from [1].

The remainder of the paper is organized in five sections. Section 2 presents some basic definitions and concepts of fuzzy theory and optimization theory. Section 3 introduces the FMFFCP, analyzes its formulation, and deals with its non-linearity. Section 4 organizes the goal programming based solution approach proposed for the FMFFCP. Section 5 contains an illustrative numerical example from the literature to analyze the performance of the proposed solution approach provided in the previous section. Finally, the paper is ended by remarking on some conclusions in Section 6.

\section{Basic concepts}

Some basic definitions of fuzzy theory, which will be applied later in this paper, are given in this section.

Definition 1. Let $X$ be a non-empty set of elements $x$. A fuzzy set $\tilde{A}$ in $X$ is defined by a set of ordered pairs $\tilde{A}=\left\{\left(x, \mu_{\tilde{A}}(x)\right) ; x \in X\right\} . \mu_{\tilde{A}}(x): X \rightarrow[0,1]$ is the membership degree of $x$ in $\tilde{A}$ and it is called the membership function of $\tilde{A}$.

Definition 2. A triangular fuzzy number is shown by $\tilde{A}=\left(a^{1}, a^{2}, a^{3}\right)$ with the membership function as follows:

$$
\mu_{\tilde{A}}(x)= \begin{cases}0 & x \leq a^{1} \\ \frac{x-a^{1}}{a^{2}-a^{1}} & a^{1} \leq x \leq a^{2} \\ \frac{a^{3}-x}{a^{3}-a^{2}} & a^{2} \leq x \leq a^{3} \\ 0 & x \geq a^{3}\end{cases}
$$

Definition 3 [22]. A triangular fuzzy number $\tilde{A}=$ $\left(a^{1}, a^{2}, a^{3}\right)$ is said to be non-negative if $a^{1} \geq 0$.

Definition 4 [22]. Let $\tilde{A}=\left(a^{1}, a^{2}, a^{3}\right)$ and $\tilde{B}=\left(b^{1}, b^{2}, b^{3}\right)$ be two non-negative triangular fuzzy numbers. Then, the following fuzzy operators are defined: $\tilde{A} \oplus \tilde{B}=\left(a^{1}+b^{1}, a^{2}+b^{2}, a^{3}+b^{3}\right), \tilde{A} \Theta \tilde{B}=$ $\left(a^{1}-b^{3}, a^{2}-b^{2}, a^{3}+b^{1}\right), \tilde{A} \otimes \tilde{B}=\left(a^{1} b^{1}, a^{2} b^{2}, a^{3} b^{3}\right)$, and $\tilde{A} \div \tilde{B}=\left(\frac{a^{1}}{b^{3}}, \frac{a^{2}}{b^{2}}, \frac{a^{3}}{b^{1}}\right)$.

Definition 5. If $\tilde{Z}=\left(Z^{1}, Z^{2}, Z^{3}\right)$ is a triangular fuzzy objective function being calculated from triangular fuzzy parameters or variables, according to the concept of component-wise optimization (see also [23]), the following optimization problem:

$$
\min \tilde{Z}=\left(Z^{1}, Z^{2}, Z^{3}\right),
$$

is equivalent to the following multi-objective optimization problem:

$$
\left\{\begin{array}{l}
\min Z^{1} \\
\min Z^{2} \\
\min Z^{3}
\end{array}\right.
$$


Definition 6. Let $X^{*}$ be a feasible solution to Problem (3). Then, $X^{*}$ is called an efficient or a Pareto-optimal solution if there is no other feasible solution $X$ such that $Z^{i} \leq Z^{i *}, i=1,2,3$ with at least one strict inequality.

\section{Fuzzy Multi-objective Fractional Fixed Charge Problem (FMFFCP)}

As mentioned in [1], FMFFCP is formulated as follows:

$$
\min \tilde{Z}(X)=\left(\tilde{Z}_{1}(X), \tilde{Z}_{2}(X), \ldots, \tilde{Z}_{p}(X)\right),
$$

subject to:

$$
\begin{aligned}
& \sum_{j=1}^{n} a_{i j} x_{j}=b_{i} \quad i=1,2, \ldots, m \\
& x_{j} \geq 0 \quad j=1,2, \ldots, n
\end{aligned}
$$

where, $p$ is the number of objective functions each of which is defined as follows:

$$
\tilde{Z}_{r}(X)=\frac{\sum_{j=1}^{n} \tilde{c}_{r j} x_{j}+\sum_{j=1}^{n} \tilde{F}_{r j}}{\sum_{j=1}^{n} \tilde{d}_{r j} x_{j}+\sum_{j=1}^{n} \tilde{f}_{r j}+\tilde{\gamma}_{r}} \quad r=1,2, \ldots, p .
$$

In the above formula, $\tilde{c}_{r j}$ and $\tilde{d}_{r j}$ are triangular fuzzy variable charges for the variable $x_{j}$, while $\tilde{F}_{r j}$ and $\tilde{f}_{r j}$ are triangular fuzzy fixed charges for the variable $x_{j}$. Since for each objective function, the condition $\sum_{j=1}^{n} \tilde{d}_{r j} x_{j}+\sum_{j=1}^{n} \tilde{f}_{r j}+\tilde{\gamma}_{r}>0$ is assumed, $\tilde{\gamma}_{r}$ is a nonnegative triangular fuzzy parameter.

Each of the fixed charges $\tilde{F}_{r j}$ and $\tilde{f}_{r j}$ is of a step function (see [24-26]) with $l$ steps defined as follows:

$$
\begin{aligned}
& \tilde{F}_{r j}=\sum_{k=1}^{l} \tilde{G}_{r j k} \delta_{r j k} \quad r=1,2, \ldots, p, \quad j=1,2, \ldots, n, \\
& \tilde{f}_{r j}=\sum_{k=1}^{l} \tilde{g}_{r j k} \eta_{r j k} \quad r=1,2, \ldots, p, \quad j=1,2, \ldots, n .
\end{aligned}
$$

In the above step functions, $\delta_{r j k}$ and $\eta_{r j k}$ are binary variables defined as follows:

$$
\begin{aligned}
& \delta_{r j k}= \begin{cases}1 & \text { if } A_{r j, k-1}<x_{j} \leq A_{r j k} \\
0 & \text { otherwise }\end{cases} \\
& \eta_{r j k}= \begin{cases}1 & \text { if } B_{r j, k-1}<x_{j} \leq B_{r j k} \\
0 & \text { otherwise }\end{cases}
\end{aligned}
$$

where $A_{r j 0}, A_{r j 1}, \ldots, A_{r j l}$ (with the condition $0=$ $\left.A_{r j 0}<A_{r j 1}<\ldots<A_{r j l}\right)$ and $B_{r j 0}, B_{r j 1}, \ldots, B_{r j l}$, (with the condition $0=B_{r j 0}<B_{r j 1}<\ldots<B_{r j l}$ ) are constant values, which determine the limits of fixed charges. Notably, the values of $\tilde{G}_{r j k}, \tilde{g}_{r j k}, A_{r j k}$, and $B_{r j k}$ are defined by the decision maker.
Considering Eqs. (5)-(9), the FMFFCP formulated in Eq. (4) is reformulated by Model (10)-(15) in which the set of Constraints (12)-(14) is equivalent to the set of Constraints (8) and (9).

$$
\min \tilde{Z}(X)=\left(\tilde{Z}_{1}(X), \tilde{Z}_{2}(X), \ldots, \tilde{Z}_{p}(X)\right),
$$

subject to:

$$
\begin{gathered}
\sum_{j=1}^{n} a_{i j} x_{j}=b_{i} \quad i=1,2, \ldots, m, \\
\delta_{r j k}= \begin{cases}1 & \text { if } A_{r j, k-1}<x_{j} \leq A_{r j k} \\
0 & \text { otherwise }\end{cases} \\
\quad r=1,2, \ldots, p, \quad j=1,2, \ldots, n, \quad k=1,2, \ldots, l, \\
\eta_{r j k}= \begin{cases}1 & \text { if } B_{r j, k-1}<x_{j} \leq B_{r j k} \\
0 & \text { otherwise }\end{cases} \\
\quad r=1,2, \ldots, p, \quad j=1,2, \ldots, n, \quad k=1,2, \ldots, l, \\
\delta_{r j k}, \eta_{r j k} \in\left\{\begin{array}{l}
0,1\} \\
r
\end{array}=1,2, \ldots, p, \quad j=1,2, \ldots, n, \quad k=1,2, \ldots, l,\right. \\
x_{j} \geq 0 \quad j=1,2, \ldots, n,
\end{gathered}
$$

where:

$$
\begin{gathered}
\tilde{Z}_{r}(X)=\frac{\sum_{j=1}^{n} \tilde{c}_{r j} x_{j}+\sum_{j=1}^{n} \sum_{k=1}^{l} \tilde{G}_{r j k} \delta_{r j k}}{\sum_{j=1}^{n} \tilde{d}_{r j} x_{j}+\sum_{j=1}^{n} \sum_{k=1}^{l} \tilde{g}_{r j k} \eta_{r j k}+\tilde{\gamma}_{r}} \\
r=1,2, \ldots, p .
\end{gathered}
$$

In the next section of the paper, an effective solution approach is proposed for solving Model (10)-(15) to obtain a Pareto-optimal solution.

\section{Proposed solution approach}

The FMFFCP formulated by Model (10)-(15) is solved in this section. Before starting to explain the methodology of the proposed approach, having a look at the only previous study focusing on the FMFFCP is necessary. As mentioned earlier, Upmanyu and Saxena [1] proposed an approach for solving the FMFFCP. In their proposed approach, a critical error exists that affects all steps and, in most of cases, gives a wrong solution. In summary, the approach applies a fuzzy ranking function in an absolutely wrong way. The details of the errors of the approach can be found in a commentary written by Kaur and Kumar [2]. Therefore, to tackle the FMFFCP in an effective way for obtaining a good 


$$
\tilde{Z}_{r}(X)=\frac{\sum_{j=1}^{n}\left(c_{r j}^{1}, c_{r j}^{2}, c_{r j}^{3}\right) x_{j}+\sum_{j=1}^{n} \sum_{k=1}^{l}\left(G_{r j k}^{1}, G_{r j k}^{2}, G_{r j k}^{3}\right) \delta_{r j k}}{\sum_{j=1}^{n}\left(d_{r j}^{1}, d_{r j}^{2}, d_{r j}^{3}\right) x_{j}+\sum_{j=1}^{n} \sum_{k=1}^{l}\left(g_{r j k}^{1}, g_{r j k}^{2}, g_{r j k}^{3}\right) \eta_{r j k}+\left(\gamma_{r}^{1}, \gamma_{r}^{2}, \gamma_{r}^{3}\right)} \quad r=1,2, \ldots, p
$$

Box I

$$
\tilde{Z}_{r}(X)=\frac{\left(\sum_{j=1}^{n} c_{r j}^{1} x_{j}+\sum_{j=1}^{n} \sum_{k=1}^{l} G_{r j k}^{1} \delta_{r j k}, \sum_{j=1}^{n} c_{r j}^{2} x_{j}+\sum_{j=1}^{n} \sum_{k=1}^{l} G_{r j k}^{2} \delta_{r j k}, \sum_{j=1}^{n} c_{r j}^{3} x_{j}+\sum_{j=1}^{n} \sum_{k=1}^{l} G_{r j k}^{3} \delta_{r j k}\right)}{\left(\sum_{j=1}^{n} d_{r j}^{1} x_{j}+\sum_{j=1}^{n} \sum_{k=1}^{l} g_{r j k}^{1} \eta_{r j k}+\gamma_{r}^{1}, \sum_{j=1}^{n} d_{r j}^{2} x_{j}+\sum_{j=1}^{n} \sum_{k=1}^{l} g_{r j k}^{2} \eta_{r j k}+\gamma_{r}^{2}, \sum_{j=1}^{n} d_{r j}^{3} x_{j}+\sum_{j=1}^{n} \sum_{k=1}^{l} g_{r j k}^{3} \eta_{r j k}+\gamma_{r}^{3}\right)}
$$

$r=1,2, \ldots, p$.

\section{Box II}

Pareto-optimal solution, a goal programming based solution approach is proposed in this section.

The FMFFCP formulated by Model (10)-(15) has two core difficulties to be solved, which are (1) its multi-objective nature and (2) its non-linear nature arising from the fractional multi-objective functions. To overcome these difficulties, a solution approach is proposed and detailed here. The proposed approach is explained in the following steps:

Step 1. As the objective functions of the FMFFCP contain triangular fuzzy parameters, expand them by Eq. (17) as shown in Box I. They are more expanded by Eq. (18) as shown in Box II. Now, based on the division operation of fuzzy numbers, Eq. (18) is converted to the triangular fuzzy value (Eq. (19)) as shown in Box III. In summary, objective function (16) is converted to objective function (19).

Step 2. Using the concept of component-wise optimization (explained in Section 2), reformulate the FMFFCP as the following multi-objective problem:

$$
\begin{gathered}
\min Z_{r}^{1}(X)=\frac{\sum_{j=1}^{n} c_{r j}^{1} x_{j}+\sum_{j=1}^{n} \sum_{k=1}^{l} G_{r j k}^{1} \delta_{r j k}}{\sum_{j=1}^{n} d_{r j}^{3} x_{j}+\sum_{j=1}^{n} \sum_{k=1}^{l} g_{r j k}^{3} \eta_{r j k}+\gamma_{r}^{3}} \\
r=1,2, \ldots, p,
\end{gathered}
$$

$\min Z_{r}^{2}(X)=\frac{\sum_{j=1}^{n} c_{r j}^{2} x_{j}+\sum_{j=1}^{n} \sum_{k=1}^{l} G_{r j k}^{2} \delta_{r j k}}{\sum_{j=1}^{n} d_{r j}^{2} x_{j}+\sum_{j=1}^{n} \sum_{k=1}^{l} g_{r j k}^{2} \eta_{r j k}+\gamma_{r}^{2}}$

$r=1,2, \ldots, p$,

$\min Z_{r}^{3}(X)=\frac{\sum_{j=1}^{n} c_{r j}^{3} x_{j}+\sum_{j=1}^{n} \sum_{k=1}^{l} G_{r j k}^{3} \delta_{r j k}}{\sum_{j=1}^{n} d_{r j}^{1} x_{j}+\sum_{j=1}^{n} \sum_{k=1}^{l} g_{r j k}^{1} \eta_{r j k}+\gamma_{r}^{1}}$

$r=1,2, \ldots, p$,

$\tilde{Z}_{r}(X)=\left(\frac{\sum_{j=1}^{n} c_{r j}^{1} x_{j}+\sum_{j=1}^{n} \sum_{k=1}^{l} G_{r j k}^{1} \delta_{r j k}}{\sum_{j=1}^{n} d_{r j}^{3} x_{j}+\sum_{j=1}^{n} \sum_{k=1}^{l} g_{r j k}^{3} \eta_{r j k}+\gamma_{r}^{3}}, \frac{\sum_{j=1}^{n} c_{r j}^{2} x_{j}+\sum_{j=1}^{n} \sum_{k=1}^{l} G_{r j k}^{2} \delta_{r j k}}{\sum_{j=1}^{n} d_{r j}^{2} x_{j}+\sum_{j=1}^{n} \sum_{k=1}^{l} g_{r j k}^{2} \eta_{r j k}+\gamma_{r}^{2}}, \frac{\sum_{j=1}^{n} c_{r j}^{3} x_{j}+\sum_{j=1}^{n} \sum_{k=1}^{l} G_{r j k}^{3} \delta_{r j k}}{\sum_{j=1}^{n} d_{r j}^{1} x_{j}+\sum_{j=1}^{n} \sum_{k=1}^{l} g_{r j k}^{1} \eta_{r j k}+\gamma_{r}^{1}}\right)$

$r=1,2, \ldots, p$. 
subject to:

$$
\begin{aligned}
& \sum_{j=1}^{n} a_{i j} x_{j}=b_{i}, \quad i=1,2, \ldots, m, \\
& \delta_{r j k}= \begin{cases}1 & \text { if } A_{r j, k-1}<x_{j} \leq A_{r j k} \\
0 & \text { otherwise }\end{cases} \\
& \quad r=1,2, \ldots, p, \quad j=1,2, \ldots, n, \quad k=1,2, \ldots, l, \\
& \eta_{r j k}= \begin{cases}1 & \text { if } B_{r j, k-1}<x_{j} \leq B_{r j k} \\
0 & \text { otherwise }\end{cases} \\
& \quad r=1,2, \ldots, p, \quad j=1,2, \ldots, n, \quad k=1,2, \ldots, l \\
& \delta_{r j k}, \eta_{r j k} \in\{0,1\} \\
& \quad r=1,2, \ldots, p, \quad j=1,2, \ldots, n, \quad k=1,2, \ldots, l, \\
& x_{j} \geq 0 \quad j=1,2, \ldots, n .
\end{aligned}
$$

Eqs. (20)-(27) contain $3 p$ objective functions as there are originally $p$ triangular fuzzy objective functions in the FMFFCP and, according to the procedure of this step, each one is converted to three objective functions.

Step 3. Obtain a goal for each objective function of Eqs. (20)-(27) by solving the following models. The goal for the objective function $Z_{r}^{1}(X)$ is shown by $z_{r}^{1 *}$ and so on:

$$
\begin{gathered}
z_{r}^{1 *}=\left\{\begin{array}{l}
\min Z_{r}^{1}(X)=\frac{\sum_{j=1}^{n} c_{r j}^{1} x_{j}+\sum_{j=1}^{n} \sum_{k=1}^{l} G_{r j k}^{1} \delta_{r j k}}{\sum_{j=1}^{n} d_{r j}^{3} x_{j}+\sum_{j=1}^{n} \sum_{k=1}^{l} g_{r j k}^{3} \eta_{r j k}+\gamma_{r}^{3}} \\
\text { Subject to Constraints }(23)-(27)
\end{array}\right. \\
r=1,2, \ldots, p \\
z_{r}^{2 *}=\left\{\begin{array}{l}
\min Z_{r}^{2}(X)=\frac{\sum_{j=1}^{n} c_{r j}^{2} x_{j}+\sum_{j=1}^{n} \sum_{k=1}^{l} G_{r j k}^{2} \delta_{r j k}}{\sum_{j=1}^{n} d_{r j}^{2} x_{j}+\sum_{j=1}^{n} \sum_{k=1}^{l} g_{r j k}^{2} \eta_{r j k}+\gamma_{r}^{2}} \\
\operatorname{Subject~to~Constraints}(23)-(27)
\end{array}\right. \\
r=1,2, \ldots, p, \\
z_{r}^{3 *}=\left\{\begin{array}{l}
\sum_{j=1}^{n} c_{r j}^{3} x_{j}+\sum_{j=1}^{n} \sum_{k=1}^{l} G_{r j k}^{3} \delta_{r j k} \\
\min Z_{r}^{3}(X)=\frac{\sum_{j=1}^{n} d_{r j}^{1} x_{j}+\sum_{j=1}^{n} \sum_{k=1}^{l} g_{r j k}^{1} \eta_{r j k}+\gamma_{r}^{1}}{\text { Subject to Constraints }(23)-(27)}
\end{array}\right. \\
r=1,2, \ldots, p .
\end{gathered}
$$

For obtaining the goals, as there are $3 p$ objective functions in the multi-objective model of Step 2, in this step, $3 p$ goals should be obtained. Hence, $3 p$ individual models should be solved, which are summarized in Models (28)-(30). It is notable that when any of Models (28)-(30) is solved for a given objective function, say $r$, Constraint sets (23)-(27), which are originally related to all $r \in\{1,2, \ldots, p\}$, consider only the given $r$, not all of them.

To linearize Models (28)-(30), the non-linear Constraints (24) and (25) for any given combination of $r, j, k$ are linearized as the constraints $x_{j}-$ $A_{r j, k-1} \leq M \delta_{r j k}$ and $x_{j}-B_{r j, k-1} \leq M \eta_{r j k}$, respectively, where $M$ is a large positive value lower bounded by $\max _{r, j}\left\{A_{r j l}-A_{r j 1}, B_{r j l}-B_{r j 1}\right\}$. Furthermore, each of Models (28)-(30) is a crisp formulation with fractional objective function. The fractional objective function can be linearized with the help of the method introduced by Charnes and Cooper [27]. To show this linearization procedure, as an example, Model (28) is linearized here. The others follow a similar procedure.

To linearize Model (28), first, the conversion $\sum_{j=1}^{n} d_{r j}^{3} x_{j}+\sum_{j=1}^{n} \sum_{k=1}^{l} g_{r j k}^{3} \eta_{r j k}+\gamma_{r}^{3}=\frac{1}{T}$ is applied, where $T$ is a continuous variable $(T>0)$. Therefore, Model (28) for its $r$-the objective function is converted to the following non-linear model (both sides of Constraints (23) and (27) and the linearized form of Constraints (24) and (25) are multiplied by $T$ ),

$$
\min Z_{r}^{1}(X)=\sum_{j=1}^{n} c_{r j}^{1} x_{j} T+\sum_{j=1}^{n} \sum_{k=1}^{l} G_{r j k}^{1} \delta_{r j k} T
$$

subject to:

$$
\begin{aligned}
& \sum_{j=1}^{n} d_{r j}^{3} x_{j} T+\sum_{j=1}^{n} \sum_{k=1}^{l} g_{r j k}^{3} \eta_{r j k} T+\gamma_{r}^{3} T=1, \\
& \sum_{j=1}^{n} a_{i j} x_{j} T=b_{i} T \quad i=1,2, \ldots, m, \\
& x_{j} T-A_{r j, k-1} T \leq M \delta_{r j k} T \\
& \quad j=1,2, \ldots, n, \quad k=1,2, \ldots, l, \\
& x_{j} T-B_{r j, k-1} T \leq M \eta_{r j k} T \\
& \quad j=1,2, \ldots, n, \quad k=1,2, \ldots, l, \\
& \delta_{r j k}, \eta_{r j k} \in\{0,1\} \quad j=1,2, \ldots, n, \quad k=1,2, \ldots, l, \\
& x_{j} T \geq 0 \quad j=1,2, \ldots, n .
\end{aligned}
$$

To linearize Problem (31), some conversions are needed. To this aim, the new variables $Y_{j}=x_{j} T$, 
$\Delta_{r j k}=\delta_{r j k} T$, and $\Omega_{r j k}=\eta_{r j k} T$ are defined. Then, Problem (31) is converted into the following:

$$
\min Z_{r}^{1}(X)=\sum_{j=1}^{n} c_{r j}^{1} Y_{j}+\sum_{j=1}^{n} \sum_{k=1}^{l} G_{r j k}^{1} \Omega_{r j k},
$$

subject to:

$$
\begin{aligned}
& \sum_{j=1}^{n} d_{r j}^{3} Y_{j}+\sum_{j=1}^{n} \sum_{k=1}^{l} g_{r j k}^{3} \Omega_{r j k}+\gamma_{r}^{3} T=1, \\
& \sum_{j=1}^{n} a_{i j} Y_{j}=b_{i} T \quad i=1,2, \ldots, m, \\
& Y_{j}-A_{r j, k-1} T \leq M \Delta_{r j k} \\
& j=1,2, \ldots, n, \quad k=1,2, \ldots, l, \\
& Y_{j}-B_{r j, k-1} T \leq M \Omega_{r j k} \\
& j=1,2, \ldots, n, \quad k=1,2, \ldots, l, \\
& \Delta_{r j k} \leq M \delta_{r j k} \\
& j=1,2, \ldots, n, \quad k=1,2, \ldots, l, \\
& \Delta_{r j k} \geq T-M\left(1-\delta_{r j k}\right) \\
& j=1,2, \ldots, n, \quad k=1,2, \ldots, l, \\
& j=1,2, \ldots, n, \quad k=1,2, \ldots, l, \\
& \Omega_{r j k} \leq T \quad j=1,2, \ldots, n, \quad k=1,2, \ldots, l, \\
& \Omega_{r j k} \geq T-M\left(1-\eta_{r j k}\right) \\
& j=1,2, \ldots, n, \quad k=1,2, \ldots, l, \\
& \Omega_{r j k} \geq 0 \quad j=1,2, \ldots, n, \quad k=1,2, \ldots, l, \\
& \delta_{r j k}, \eta_{r j k} \in\{0,1\} \\
& j=1,2, \ldots, n, \quad k=1,2, \ldots, l, \\
& Y_{j} \geq 0, \quad T>0 \quad j=1,2, \ldots, n .
\end{aligned}
$$

Model (32)-(46) is a linearized version of Model (28), where Constraints (37)-(40) are equivalent to the term $\Delta_{r j k}=\delta_{r j k} T$ and Constraints (41)-(44) are equivalent to the term $\Omega_{r j k}=\eta_{r j k} T$. These sets of constraints allow $\Delta_{r j k} \in\{0, T\}$ and $\Omega_{r j k} \in$ $\{0, T\}$. The other non-linear problems from the set of Problems (28)-(30) are linearized in the same way.

Step 4. After obtaining the goal of each objective function, we go back to solve Model (10)-(15). As, Model (10)-(15) was reformulated to Model (20)(27), we introduce an effective goal programming approach for solving the reformulated model (for more information about goal programming, see [2832]). Thus, considering the goals of each objective function obtained in Step 3, Model (20)-(27) is converted to the following non-linear formulation:

$$
\min \sum_{r=1}^{p} D_{r}^{1+}+\sum_{r=1}^{p} D_{r}^{2+}+\sum_{r=1}^{p} D_{r}^{3+},
$$

subject to:

$$
\begin{aligned}
& \frac{\sum_{j=1}^{n} c_{r j}^{1} x_{j}+\sum_{j=1}^{n} \sum_{k=1}^{l} G_{r j k}^{1} \delta_{r j k}}{\sum_{j=1}^{n} d_{r j}^{3} x_{j}+\sum_{j=1}^{n} \sum_{k=1}^{l} g_{r j k}^{3} \eta_{r j k}+\gamma_{r}^{3}}+D_{r}^{1-}-D_{r}^{1+}=z_{r}^{1 *} \\
& r=1,2, \ldots, p, \\
& \frac{\sum_{j=1}^{n} c_{r j}^{2} x_{j}+\sum_{j=1}^{n} \sum_{k=1}^{l} G_{r j k}^{2} \delta_{r j k}}{\sum_{j=1}^{n} d_{r j}^{2} x_{j}+\sum_{j=1}^{n} \sum_{k=1}^{l} g_{r j k}^{2} \eta_{r j k}+\gamma_{r}^{2}}+D_{r}^{2-}-D_{r}^{2+}=z_{r}^{2 *} \\
& r=1,2, \ldots, p, \\
& \frac{\sum_{j=1}^{n} c_{r j}^{3} x_{j}+\sum_{j=1}^{n} \sum_{k=1}^{l} G_{r j k}^{3} \delta_{r j k}}{\sum_{j=1}^{n} d_{r j}^{1} x_{j}+\sum_{j=1}^{n} \sum_{k=1}^{l} g_{r j k}^{1} \eta_{r j k}+\gamma_{r}^{1}}+D_{r}^{3-}-D_{r}^{3+}=z_{r}^{3 *} \\
& r=1,2, \ldots, p, \\
& D_{r}^{1-} D_{r}^{1+}=0 \quad r=1,2, \ldots, p, \\
& D_{r}^{2-} D_{r}^{2+}=0 \quad r=1,2, \ldots, p, \\
& D_{r}^{3-} D_{r}^{3+}=0 \quad r=1,2, \ldots, p, \\
& D_{r}^{1-}, D_{r}^{2-}, D_{r}^{3-}, D_{r}^{1+}, D_{r}^{2+}, D_{r}^{3+} \geq 0 \\
& r=1,2, \ldots, p,
\end{aligned}
$$

Constraints (23)-(27),

where $D_{r}^{1-}$ and $D_{r}^{1+}$ are negative and positive deviations of objective function $Z_{r}^{1}(X)$ from its goal $z_{r}^{1 *}$, respectively. The following issues are of interest about Eqs. (47)-(55): 
- As the objective functions are of minimization type, an objective function can never be less than its goal. Therefore, $D_{r}^{1-}=D_{r}^{2-}=D_{r}^{3-}=0$ $(\forall r \in\{1,2, \ldots, p\})$. As a result, Constraints (51)(53) can be removed from the model.

- As $D_{r}^{1-}=D_{r}^{2-}=D_{r}^{3-}=0(\forall r \in\{1,2, \ldots, p\})$, the variables $D_{r}^{1-}, D_{r}^{2-}, D_{r}^{3-}$ are removed from the Constraints (48)-(50). As it is difficult to linearize the non-linearity of these constraints, a typical variable changing technique is proposed here. To this aim, the variables are converted to the fractions:

$$
\begin{gathered}
\frac{R_{r}^{1+}}{\sum_{j=1}^{n} d_{r j}^{3} x_{j}+\sum_{j=1}^{n} \sum_{k=1}^{l} g_{r j k}^{3} \eta_{r j k}+\gamma_{r}^{3}}, \\
\frac{R_{r}^{2+}}{\sum_{j=1}^{n} d_{r j}^{2} x_{j}+\sum_{j=1}^{n} \sum_{k=1}^{l} g_{r j k}^{2} \eta_{r j k}+\gamma_{r}^{2}},
\end{gathered}
$$

and:

$$
\frac{R_{r}^{3+}}{\sum_{j=1}^{n} d_{r j}^{1} x_{j}+\sum_{j=1}^{n} \sum_{k=1}^{l} g_{r j k}^{1} \eta_{r j k}+\gamma_{r}^{1}},
$$

respectively, where the non-negative variables $R_{r}^{1+}, R_{r}^{2+}$, and $R_{r}^{3+}$ are used instead of the variables $D_{r}^{1+}, D_{r}^{2+}$, and $D_{r}^{3+}$ in objective function (47).

Considering the above issues, Constraints (48)(50) are reformulated as follows:

$$
\begin{gathered}
\frac{\sum_{j=1}^{n} c_{r j}^{1} x_{j}+\sum_{j=1}^{n} \sum_{k=1}^{l} G_{r j k}^{1} \delta_{r j k}-R_{r}^{1+}}{\sum_{j=1}^{n} d_{r j}^{3} x_{j}+\sum_{j=1}^{n} \sum_{k=1}^{l} g_{r j k}^{3} \eta_{r j k}+\gamma_{r}^{3}}=z_{r}^{1 *} \\
r=1,2, \ldots, p, \\
\frac{\sum_{j=1}^{n} c_{r j}^{2} x_{j}+\sum_{j=1}^{n} \sum_{k=1}^{l} G_{r j k}^{2} \delta_{r j k}-R_{r}^{2+}}{\sum_{j=1}^{n} d_{r j}^{2} x_{j}+\sum_{j=1}^{n} \sum_{k=1}^{l} g_{r j k}^{2} \eta_{r j k}+\gamma_{r}^{2}}=z_{r}^{2 *} \\
r=1,2, \ldots, p, \\
\frac{\sum_{j=1}^{n} c_{r j}^{3} x_{j}+\sum_{j=1}^{n} \sum_{k=1}^{l} G_{r j k}^{3} \delta_{r j k}-R_{r}^{3+}}{\sum_{j=1}^{n} d_{r j}^{1} x_{j}+\sum_{j=1}^{n} \sum_{k=1}^{l} g_{r j k}^{1} \eta_{r j k}+\gamma_{r}^{1}}=z_{r}^{3 *} \\
r=1,2, \ldots, p .
\end{gathered}
$$

Therefore, Model (45)-(55) is linearized as follows:

$$
\min \sum_{r=1}^{p} R_{r}^{1+}+\sum_{r=1}^{p} R_{r}^{2+}+\sum_{r=1}^{p} R_{r}^{3+}
$$

subject to:

$$
\begin{aligned}
& \sum_{j=1}^{n} c_{r j}^{1} x_{j}+\sum_{j=1}^{n} \sum_{k=1}^{l} G_{r j k}^{1} \delta_{r j k}-R_{r}^{1+} \\
& =z_{r}^{1 *}\left(\sum_{j=1}^{n} d_{r j}^{3} x_{j}+\sum_{j=1}^{n} \sum_{k=1}^{l} g_{r j k}^{3} \eta_{r j k}+\gamma_{r}^{3}\right) \\
& \quad r=1,2, \ldots, p
\end{aligned}
$$$$
\sum_{j=1}^{n} c_{r j}^{2} x_{j}+\sum_{j=1}^{n} \sum_{k=1}^{l} G_{r j k}^{2} \delta_{r j k}-R_{r}^{2+}
$$$$
=z_{r}^{2 *}\left(\sum_{j=1}^{n} d_{r j}^{2} x_{j}+\sum_{j=1}^{n} \sum_{k=1}^{l} g_{r j k}^{2} \eta_{r j k}+\gamma_{r}^{2}\right)
$$$$
r=1,2, \ldots, p,
$$

$$
\begin{aligned}
& \sum_{j=1}^{n} c_{r j}^{3} x_{j}+\sum_{j=1}^{n} \sum_{k=1}^{l} G_{r j k}^{3} \delta_{r j k}-R_{r}^{3+} \\
& =z_{r}^{3 *}\left(\sum_{j=1}^{n} d_{r j}^{1} x_{j}+\sum_{j=1}^{n} \sum_{k=1}^{l} g_{r j k}^{1} \eta_{r j k}+\gamma_{r}^{1}\right) \\
& r=1,2, \ldots, p, \\
& R_{r}^{1+}, R_{r}^{2+}, R_{r}^{3+} \geq 0 \quad r=1,2, \ldots, p,
\end{aligned}
$$

Constraints (21)-(26).

Finally, solving Model (59)-(64) will result in a solution to Model (10)-(15). The obtained solution may be either a Pareto-optimal solution or only a satisfactory solution to Model (10)-(15). Accordingly, a Pareto-optimality test is proposed in the next step.

Step 5. To test whether or not the solution obtained by Model (59)-(64) is a Pareto-optimal solution to Model (10)-(15), consider the following model and the consequent theorem. Notably, the solution obtained by Model (59)-(64) is supplied in Model (65)-(69). Therefore, except for the notation $R_{r}^{1+}, R_{r}^{2+}, R_{r}^{3+}$, the other notation is constant here:

$$
\max \sum_{r=1}^{p} R_{r}^{1+}+\sum_{r=1}^{p} R_{r}^{2+}+\sum_{r=1}^{p} R_{r}^{3+}
$$

subject to: 


$$
\begin{aligned}
& \sum_{j=1}^{n} c_{r j}^{1} x_{j}+\sum_{j=1}^{n} \sum_{k=1}^{l} G_{r j k}^{1} \delta_{r j k}+R_{r}^{1+} \\
& =z_{r}^{1 *}\left(\sum_{j=1}^{n} d_{r j}^{3} x_{j}+\sum_{j=1}^{n} \sum_{k=1}^{l} g_{r j k}^{3} \eta_{r j k}+\gamma_{r}^{3}\right) \\
& r=1,2, \ldots, p, \\
& \sum_{j=1}^{n} c_{r j}^{2} x_{j}+\sum_{j=1}^{n} \sum_{k=1}^{l} G_{r j k}^{2} \delta_{r j k}+R_{r}^{2+} \\
& =z_{r}^{2 *}\left(\sum_{j=1}^{n} d_{r j}^{2} x_{j}+\sum_{j=1}^{n} \sum_{k=1}^{l} g_{r j k}^{2} \eta_{r j k}+\gamma_{r}^{2}\right) \\
& r=1,2, \ldots, p, \\
& R_{r}^{1+}, R_{r}^{2+}, R_{r}^{3+} \geq 0 \\
& \sum_{j=1}^{n} c_{r j}^{3} x_{j}+\sum_{j=1}^{n} \sum_{k=1}^{l} G_{r j k}^{3} \delta_{r j k}+R_{r}^{3+} \\
& \left(\sum_{j=1}^{n} d_{r j}^{1} x_{j}+\sum_{j=1}^{n} \sum_{k=1}^{l} g_{r j k}^{1} \eta_{r j k}+\gamma_{r}^{1}\right)
\end{aligned}
$$

Theorem 1. If the optimal value of Problem (65)(69) for a solution obtained by Model (59)-(64) is zero, then the obtained solution is Pareto-optimal solution to Model (10)-(15); otherwise, it is not Pareto-optimal.

Proof. In order to simplify the proof, Problem (10)(15) is represented by the formulation below:

$$
\min Z_{r}(X)=\frac{f_{r}(x)}{g_{r}(x)} \quad r=1,2, \ldots, p,
$$

subject to:

$$
x \in S \text {, }
$$

while Problem (65)-(69) is represented by the following formulation:

$$
\max \sum_{r=1}^{p} d_{r}
$$

subject to:

$$
\begin{aligned}
& f_{r}(x)+d_{r}-z_{r}^{*} g_{r}(x)=0 \quad r=1,2, \ldots, p, \\
& x \in S \\
& d_{r} \geq 0 \quad r=1,2, \ldots, p .
\end{aligned}
$$

Now, we assume that the optimal objective function value for Problem (71) is zero and its associated optimal solution $x^{\prime}$, also obtained by Model (59)-(64), is not Pareto-optimal for Problem (10)-(15). Thus, there should be a solution, say $x^{\prime \prime}$, which is better than $x^{\prime}$ by the following condition:

$$
\frac{f_{r}\left(x^{\prime \prime}\right)}{g_{r}\left(x^{\prime \prime}\right)} \leq \frac{f_{r}\left(x^{\prime}\right)}{g_{r}\left(x^{\prime}\right)} \quad r=1,2, \ldots, p,
$$

and $\exists j \in\{1,2, \ldots, p\}$ such that:

$$
\frac{f_{j}\left(x^{\prime \prime}\right)}{g_{j}\left(x^{\prime \prime}\right)}<\frac{f_{j}\left(x^{\prime}\right)}{g_{j}\left(x^{\prime}\right)} .
$$

Considering a non-negative slack variable of $d_{r} / g_{r}\left(x^{\prime \prime}\right)$, $i=1,2, \ldots, p$ for Inequalities (72) and (73), the following equality is obtained:

$$
\begin{gathered}
\frac{f_{r}\left(x^{\prime \prime}\right)}{g_{r}\left(x^{\prime \prime}\right)}+\frac{d_{r}}{g_{r}\left(x^{\prime \prime}\right)}=\frac{f_{r}\left(x^{\prime}\right)}{g_{r}\left(x^{\prime}\right)}, \\
r=1,2, \ldots, p,
\end{gathered}
$$

where $\bar{d}=\left(d_{1}, d_{2}, \ldots, d_{p}\right) \geq 0, \bar{d} \neq 0$. If we multiply both sides of Eq. (74) by $g_{r}\left(x^{\prime \prime}\right)$, the following equation is obtained:

$$
\begin{aligned}
& f_{r}\left(x^{\prime \prime}\right)+d_{r}=\frac{f_{r}\left(x^{\prime}\right)}{g_{r}\left(x^{\prime}\right)} g_{r}\left(x^{\prime \prime}\right)=z_{r}^{\prime} g_{r}\left(x^{\prime \prime}\right) \\
& r=1,2, \ldots, p .
\end{aligned}
$$

According to Eq. (75), it is concluded that $\left(x^{\prime \prime}, \bar{d}\right)$ is a feasible solution to Problem $(71)$, where $\bar{d} \neq 0$. The value implies that there is a contradiction with the initial assumption (the optimal objective function value of Problem (71) is zero) and the related optimum objective function value must be positive. Therefore, $x^{\prime}$ is a Pareto-optimal solution to Model (71).

In order to prove the theorem conversely, first, we assume that $x^{\prime}$ is a Pareto-optimal solution to Problem (10)-(15). Therefore, it should be shown that the optimal objective function value of Problem (71) is zero. To do so, a contradiction is considered by assuming a positive value for the objective function of Problem (71). Therefore, $(\bar{x}, \bar{d})$ is a feasible solution to Problem (71) with $\bar{d} \neq 0$. As $g_{r}(\bar{x})>0, r=1, \ldots, p$, the constraints of the problem are rewritten by the following equality:

$$
\bar{Z}_{r}+\frac{\bar{d}_{r}}{g_{r}(\bar{x})}=z_{r}^{*} \quad r=1,2, \ldots, p,
$$

where $\bar{Z}_{r}=f_{r}(\bar{x}) / g_{r}(\bar{x})$. Also, the following relation can be obtained:

$$
\bar{Z}_{r}=z_{r}^{*}-\frac{\bar{d}_{r}}{g_{r}(\bar{x})}<\bar{Z}_{r}^{\prime} \quad r=1,2, \ldots, p .
$$


As a result of Relation (77), $x^{\prime}$ is not a Pareto-optimal solution to Problem (71). This claim is in contradiction with the initial assumption $\left(x^{\prime}\right.$ is a Pareto-optimal solution to Problem (10)-(15)). Therefore, the optimal value of Problem (71) must be zero.

\section{An illustrative example}

To study the performance of the proposed solution approach detailed in Steps 1-4, an example of FMFFCP from the study of Upmanyu and Saxena [1] is considered. In this example, there are two variables ( $n=2$, hence $j \in\{1,2\})$, two original constraints ( $m=$ 2 , hence $i \in\{1,2\}$ ), three objective functions ( $p=3$, hence $r \in\{1,2,3\}$ ), and three steps of fixed charges ( $l=3$, hence $k \in\{1,2,3\})$. The data for the problem is summarized in Tables 1 and 2. Notably, in Table 2 , the intervals of are obtained by the data $A_{r j 0}=0$, $A_{r j 1}=1, A_{r j 2}=2, A_{r j 3}=\infty, B_{r j 0}=0, B_{r j 1}=1$, $B_{r j 2}=2$, and $B_{r j 3}=\infty(\forall r \in\{1,2,3\}, j \in\{1,2\})$.

The original formulation of this example (Eq. (78)) is shown in Box IV. Taking Steps 1-3 of the proposed approach for Eq. (78), the goals of the goal programming formulation of Step 4 are obtained. The outputs of Step 3 on Eq. (78) and the data for the example are depicted in Table 3 . As nine problems have to be solved in Step 3, the results for each problem can be realized in the table.

Supplying the results of Table 3 to the goal programming formulations (Eqs. (59)-(64)) completes Step 4 for the example. Therefore, by solving Eqs. (59)-(64) for the data of this example, the results in Table 4 are obtained. The results contain the obtained solution and the triangular objective function values of the FMFFCP. The triangular objective functions can be extracted from Constraints (60)-(62).

It is notable that if the obtained results in Table 4 are used to test Pareto-optimality of the solution by the proposed formulation in Step 5, the objective function of Model (65)-(69) would be zero, which proves Paretooptimality of the obtained solution.

As the method proposed by Upmanyu and Saxena [1] is based on wrong mathematical concepts (see $[2])$, it cannot be effective for the case of FMFFCP.

Table 1. Some data on the example.

\begin{tabular}{lcclccc}
\hline & \multicolumn{2}{c}{$\tilde{\boldsymbol{c}}_{\boldsymbol{r} \boldsymbol{j}}$} & & \multicolumn{2}{c}{$\tilde{\boldsymbol{d}}_{\boldsymbol{r} \boldsymbol{j}}$} & $\tilde{\boldsymbol{\gamma}}_{\boldsymbol{r}}$ \\
\cline { 2 - 3 } & $\boldsymbol{j}=\mathbf{1}$ & $\boldsymbol{j}=\mathbf{2}$ & & $\boldsymbol{j}=\mathbf{1}$ & $\boldsymbol{j}=\mathbf{2}$ & \\
\hline $\boldsymbol{r}=\mathbf{1}$ & $(7,8,9)$ & $(1,5,11)$ & & $(2,4,7)$ & $(5,6,7)$ & $(4,8,12)$ \\
$\boldsymbol{r}=\mathbf{2}$ & $(3,6,7)$ & $(4,8,15)$ & & $(3,7,9)$ & $(7,9,13)$ & $(0,1,2)$ \\
$\boldsymbol{r}=\mathbf{3}$ & $(1,3,5)$ & $(1,4,9)$ & & $(1,2,3)$ & $(1,3,9)$ & $(0,2,4)$ \\
\hline
\end{tabular}

Table 2. Some data on the example.

\begin{tabular}{|c|c|c|c|c|c|c|c|c|}
\hline & & & \multicolumn{2}{|c|}{$r=1$} & \multicolumn{2}{|c|}{$r=2$} & \multicolumn{2}{|c|}{$r=3$} \\
\hline & & & $\tilde{G}_{r j l}$ & $\tilde{\boldsymbol{g}}_{r j k}$ & $\tilde{G}_{r j l}$ & $\tilde{\boldsymbol{g}}_{r j k}$ & $\tilde{G}_{r j l}$ & $\tilde{\boldsymbol{g}}_{r j k}$ \\
\hline \multirow{3}{*}{$j=1$} & $k=1$ & $0<x_{j} \leq 1$ & $(20,22,27)$ & $(19,21,28)$ & $(15,16,17)$ & $(12,14,16)$ & $(10,13,17)$ & $(16,18,20)$ \\
\hline & $k=2$ & $1<x_{j} \leq 2$ & $(24,25,26)$ & $(20,27,29)$ & $(14,18,22)$ & $(12,13,15)$ & $(12,17,18)$ & $(16,20,21)$ \\
\hline & $k=3$ & $2<x_{j}$ & $(21,23,25)$ & $(25,26,27)$ & $(11,13,15)$ & $(13,17,19)$ & $(14,17,20)$ & $(17,19,22)$ \\
\hline \multirow{3}{*}{$j=2$} & $k=1$ & $0<x_{j} \leq 1$ & $(14,18,22)$ & $(10,13,17)$ & $(16,20,21)$ & $(24,25,26)$ & $(13,17,19)$ & $(20,27,29)$ \\
\hline & $k=2$ & $1<x_{j} \leq 2$ & $(11,13,15)$ & $(14,17,20)$ & $(17,19,22)$ & $(20,22,27)$ & $(12,13,15)$ & $(25,26,27)$ \\
\hline & $k=3$ & $2<x_{j}$ & $(15,16,17)$ & $(12,17,18)$ & $(16,18,20)$ & $(21,23,25)$ & $(12,14,16)$ & $(19,21,28)$ \\
\hline
\end{tabular}

Table 3. The goals and their related solutions for the example.

\begin{tabular}{cccc}
\hline & $\boldsymbol{z}_{\boldsymbol{r}}^{\mathbf{1 *}},\left(\boldsymbol{x}_{\mathbf{1}}^{*}, \boldsymbol{x}_{\mathbf{2}}^{*}, \boldsymbol{T}^{*}\right)$ & $\boldsymbol{z}_{\boldsymbol{r}}^{\mathbf{2 *}},\left(\boldsymbol{x}_{\mathbf{1}}^{*}, \boldsymbol{x}_{\mathbf{2}}^{*}, \boldsymbol{T}^{*}\right)$ & $\boldsymbol{z}_{\boldsymbol{r}}^{\mathbf{3}},\left(\boldsymbol{x}_{\mathbf{1}}^{*}, \boldsymbol{x}_{\mathbf{2}}^{*}, \boldsymbol{T}^{*}\right)$ \\
\hline$r=1$ & $0.583,(1.14,2.57,0.008)$ & $0.938,(2,0,0.012)$ & $1.29,(2,0,0.012)$ \\
$r=2$ & $0.514,(3.5,1,0.11)$ & $0.893,(5,0,0.007)$ & $1.133,(2,0,0.012)$ \\
$r=3$ & $0.409,(5,0,0.015)$ & $0.678,(2,0,0.008)$ & $0.935,(2,0,0.016)$ \\
\hline
\end{tabular}

Table 4. Pareto-optimal solution obtained by the proposed approach for the example.

\begin{tabular}{ccccc}
\hline$\tilde{\boldsymbol{Z}}_{\mathbf{1}}(\boldsymbol{X})$ & $\tilde{\boldsymbol{Z}}_{\mathbf{2}}(\boldsymbol{X})$ & $\tilde{\boldsymbol{Z}}_{\mathbf{3}}(\boldsymbol{X})$ & $\boldsymbol{x}_{\mathbf{1}}$ & $\boldsymbol{x}_{\boldsymbol{2}}$ \\
\hline$(0.670,0.938,1.410)$ & $(0.667,1.016,1.5)$ & $(0.462,0.7,1.017)$ & 2 & 0 \\
\hline
\end{tabular}




$$
\begin{aligned}
& \tilde{Z}_{1}(X)=\frac{\tilde{c}_{11} x_{1}+\tilde{c}_{12} x_{2}+\tilde{G}_{111} \delta_{111}+\tilde{G}_{112} \delta_{112}+\tilde{G}_{113} \delta_{113}+\tilde{G}_{121} \delta_{121}+\tilde{G}_{122} \delta_{122}+\tilde{G}_{123} \delta_{123}}{\tilde{d}_{11} x_{1}+\tilde{d}_{12} x_{2}+\tilde{g}_{111} \eta_{111}+\tilde{g}_{112} \eta_{112}+\tilde{g}_{113} \eta_{113}+\tilde{g}_{121} \eta_{121}+\tilde{g}_{122} \eta_{122}+\tilde{g}_{123} \eta_{123}+\tilde{\gamma}_{1}} \\
& \tilde{Z}_{2}(X)=\frac{\tilde{c}_{21} x_{1}+\tilde{c}_{22} x_{2}+\tilde{G}_{211} \delta_{211}+\tilde{G}_{212} \delta_{212}+\tilde{G}_{213} \delta_{213}+\tilde{G}_{221} \delta_{221}+\tilde{G}_{222} \delta_{222}+\tilde{G}_{223} \delta_{223}}{\tilde{d}_{21} x_{1}+\tilde{d}_{22} x_{2}+\tilde{g}_{211} \eta_{211}+\tilde{g}_{212} \eta_{212}+\tilde{g}_{213} \eta_{213}+\tilde{g}_{221} \eta_{221}+\tilde{g}_{222} \eta_{222}+\tilde{g}_{223} \eta_{223}+\tilde{\gamma}_{2}}, \\
& \tilde{Z}_{3}(X)=\frac{\tilde{c}_{31} x_{1}+\tilde{c}_{32} x_{2}+\tilde{G}_{311} \delta_{311}+\tilde{G}_{312} \delta_{312}+\tilde{G}_{313} \delta_{313}+\tilde{G}_{321} \delta_{321}+\tilde{G}_{322} \delta_{322}+\tilde{G}_{323} \delta_{323}}{\tilde{d}_{31} x_{1}+\tilde{d}_{32} x_{2}+\tilde{g}_{311} \eta_{311}+\tilde{g}_{312} \eta_{312}+\tilde{g}_{313} \eta_{313}+\tilde{g}_{321} \eta_{321}+\tilde{g}_{322} \eta_{322}+\tilde{g}_{323} \eta_{323}+\tilde{\gamma}_{3}},
\end{aligned}
$$

subject to:

$$
\begin{aligned}
& 3 x_{1}+x_{2} \geq 6 \\
& 2 x_{1}+3 x_{2} \leq 10 \\
& x_{1}-A_{110} \leq M \delta_{111} \quad x_{2}-A_{120} \leq M \delta_{121} \quad x_{1}-B_{110} \leq M \eta_{111} \quad x_{2}-B_{120} \leq M \eta_{121} \\
& x_{1}-A_{111} \leq M \delta_{112} \quad x_{2}-A_{121} \leq M \delta_{122} \quad x_{1}-B_{111} \leq M \eta_{112} \quad x_{2}-B_{121} \leq M \eta_{122} \\
& x_{1}-A_{112} \leq M \delta_{113} \quad x_{2}-A_{122} \leq M \delta_{123} \quad x_{1}-B_{112} \leq M \eta_{113} \quad x_{2}-B_{122} \leq M \eta_{123} \\
& x_{1}-A_{210} \leq M \delta_{211} \quad x_{2}-A_{220} \leq M \delta_{221} \quad x_{1}-B_{210} \leq M \eta_{211} \quad x_{2}-B_{220} \leq M \eta_{221} \\
& x_{1}-A_{211} \leq M \delta_{212} \quad x_{2}-A_{221} \leq M \delta_{222} \quad x_{1}-B_{211} \leq M \eta_{212} \quad x_{2}-B_{221} \leq M \eta_{222} \\
& x_{1}-A_{212} \leq M \delta_{213} \quad x_{2}-A_{222} \leq M \delta_{223} \quad x_{1}-B_{212} \leq M \eta_{213} \quad x_{2}-B_{222} \leq M \eta_{223} \\
& x_{1}-A_{310} \leq M \delta_{311} \quad x_{2}-A_{320} \leq M \delta_{321} \quad x_{1}-B_{310} \leq M \eta_{311} \quad x_{2}-B_{320} \leq M \eta_{321} \\
& x_{1}-A_{311} \leq M \delta_{312} \quad x_{2}-A_{321} \leq M \delta_{322} \quad x_{1}-B_{311} \leq M \eta_{312} \quad x_{2}-B_{321} \leq M \eta_{322} \\
& x_{1}-A_{312} \leq M \delta_{313} \quad x_{2}-A_{322} \leq M \delta_{323} \quad x_{1}-B_{312} \leq M \eta_{313} \quad x_{2}-B_{322} \leq M \eta_{323} \\
& \delta_{111}, \delta_{112}, \delta_{113}, \delta_{211}, \delta_{212}, \delta_{213}, \delta_{311}, \delta_{312}, \delta_{313}, \delta_{121}, \delta_{122}, \delta_{123}, \delta_{221}, \delta_{222}, \delta_{223}, \delta_{321}, \delta_{322}, \delta_{323} \in\{0,1\}
\end{aligned}
$$$$
\eta_{111}, \eta_{112}, \eta_{113}, \eta_{211}, \eta_{212}, \eta_{213}, \eta_{311}, \eta_{312}, \eta_{313}, \eta_{121}, \eta_{122}, \eta_{123}, \eta_{221}, \eta_{222}, \eta_{223}, \eta_{321}, \eta_{322}, \eta_{323} \in\{0,1\}
$$$$
x_{1}, x_{2} \geq 0 .
$$

\section{Box IV}

That is, the method of Upmanyu and Saxena [1] cannot guarantee obtaining a good solution, especially with large-size instances. Instead, the proposed solution approach in this study tries to overcome the shortcomings of the approach of Upmanyu and Saxena [1], e.g., using ranking functions. The proposed approach does not use any ranking function, which is an important advantage for solving fuzzy problems. On the other hand, the proposed approach can easily solve large-size instances of the FMFFCP. Finally, it is notable that the goal programming in Steps 3-4 of the proposed approach is not constructed based on preferences of the decision maker.

\section{Concluding remarks}

In this study, a Fuzzy Multi-objective Fractional Fixed Charge Problem (FMFFCP) consisting of several fractional objective functions with triangular fuzzy parameters was considered. The problem had previously been tackled only by Upmanyu and Saxena [1] through a method containing wrong mathematical concepts (see the commentary of Kaur and Kumar [2]). To overcome the shortcomings of this method, a goal programming based solution approach was proposed to solve the FMFFCP for obtaining a Pareto-optimal solution. The 
proposed approach applied no ranking function of fuzzy numbers and its goal programming stage considered no preference of the decision maker. The computational experiments provided by an example from the literature proved effectiveness of the proposed approach.

\section{References}

1. Upmanyu, M. and Saxena, R.R. "On solving a multiobjective fixed charge problem with imprecise fractional objectives", Applied Soft Computing, 40, pp. 64-69 (2016).

2. Kaur, J. and Kumar, A. "Commentary on "On solving a multiobjective fixed charge problem with imprecise fractional objectives"”, Applied Soft Computing, 61, pp. 681-682 (2017).

3. Hirsch, W. and Dantzig, G.B. "The fixed charge problem", Navel Research Logistics, 15, pp. 413-424 (1968).

4. Steinberg, D.I. "The fixed charge problem", Navel Research Logistics Quarterly, 172, pp. 17-36 (1970).

5. Cooper, L. and Drebes, C. "An approximate solution method for the fixed charge problem", Navel Research Logistics, 14, pp. 101-113 (1967).

6. Murty, K.G. "Solving the fixed charge transportation problem by extreme point ranking", Operations Research Quarterly, 16, pp. 268-279 (1968).

7. Walker, W.E. "A heuristic adjacent extreme point algorithm for the fixed charge problem", Management Science, 22, pp. 587-596 (1976).

8. Adlakha, V. and Kowalski, K. "A heuristic algorithm for the fixed charge problem", Opsearch, 47(2) pp. 166-175 (2010).

9. Gendron, B. and Larose, M. "Branch-and-price-andcut for large-scale multicom-modity capacitated fixedcharge network design", EURO Journal on Computational Optimization, 2(1-2), pp. 55-75 (2014).

10. Fernández, E. and Landete, M. "Fixed-charge facility location problems", Location Science, Springer, pp. 47-77 (2015).

11. Shishebori, D., Yousefi Babadi, A., and Noormohammadzadeh, Z. "A Lagrangian relaxation approach to fuzzy robust multi-objective facility location network design problem", Scientia Iranica, 25(3), pp. 17501767 (2018).

12. Rabieh, M., Modarres, M., and Azar, A. "Robustfuzzy model for supplier selection under uncertainty: An application to the automobile industry", Scientia Iranica, 25(4), pp. 2297-2311 (2018).

13. Arora, S.R. "A note on fractional fixed charge problem", NZOR, 5(2), pp. 66-71 (1977).

14. Hong, J., Diabat, A., Panicker, V.V., and Rajagopalan, S. "A two-stage supply chain problem with fixed costs: An ant colony optimization approach", International Journal of Production Economics, 204, pp. 214-226 (2018).
15. Calvete, H.I., Galé, C., Iranzo, J.A., and Toth, P. "A metaheuristic for the two-stage fixed-charge transportation problem", Computers \& Operations Research, 95, pp. 113-122 (2018).

16. Bertazzi, L. and Maggioni, F. "A stochastic multistage fixed charge transportation problem: Worst-case analysis of the rolling horizon approach", European Journal of Operational Research, 267(2), pp. 555-569 (2018).

17. Bazaraa, M.S., Sherali, H.D., and Shetty C.M., Nonlinear Programming: Theory and Algorithms, John Wiley \& Sons, New York (2013).

18. Stancu Minasian, I.M. "A eighth bibliography of fractional programming", Optimization, 66(3), pp. 439470 (2017).

19. Almogy, Y. and Levin, O. "The fractional fixed charge problem", Navel Research Logistics, 18(3), pp. 307315 (1971).

20. Arya, R., Singh, P., and Bhati, D. "A fuzzy based branch and bound approach for multi-objective linear fractional (MOLF) optimization problems", Journal of Computational Science, 24, pp. 54-64 (2018).

21. Osman, M.S., Emam, O.E., and Elsayed, M.A. "Interactive approach for multi-level multi-objective fractional programming problems with fuzzy parameters", Beni-Suef University Journal of Basic and Applied Sciences, 7(1), pp. 139-149 (2018).

22. Kaufmann, A. and Gupta, M.M., Introduction to Fuzzy Arithmetics: Theory and Applications, Van Nostrand Reinhold, New York (1985).

23. Gupta, P. and Mehlawat, M.K. "A new possibilistic programming approach for solving fuzzy multiobjective assignment problem", IEEE Transactions on Fuzzy Systems, 22(1), pp. 16-34 (2014).

24. Mahmoodirad, A., Hassasi, H., Tohidi, Gh., and Sanei, M. "On approximation of the fully fuzzy fixed charge transportation problem", International Journal of Industrial Mathematics, 6(4), pp. 307-314 (2014).

25. Mahmoodirad, A. and Sanei, M. "Solving a multistage multi-product solid supply chain network design problem by meta-heuristics", Scientia Iranica, 23(3), pp. 1428-1440 (2016).

26. Sanei, M., Mahmoodirad, A., Niroomand, S., Jamalian, A., and Gelareh, S. "Step fixed-charge solid transportation problem: a Lagrangian relaxation heuristic approach", Computational and Applied Mathematics, 36(3), pp. 1217-1237 (2017).

27. Charnes, A. and Cooper, W.W. "Programming with linear fractional functionals", Naval Research Logistics Quarterly, 9, pp. 181-186 (1962).

28. Bal, A. and Satoglu, S.I. "A goal programming model for sustainable reverse logistics operations planning and an application", Journal of Cleaner Production, 201, pp. 1081-1091 (2018).

29. Wang, Z.J. "A goal programming approach to deriving interval weights in analytic form from interval fuzzy preference relations based on multiplicative 
consistency", Information Sciences, 462, pp. 160-181 (2018).

30. Komsiyah, S. and Centika, H.E. "A fuzzy goal programming model for production planning in furniture company", Procedia Computer Science, 135, pp. 544552 (2018).

31. Xavier, A., Costa-Freitas, M.D.B., Fragoso, R., and Rosário, M.D.S. "A regional composite indicator for analysing agricultural sustainability in Portugal: A goal programming approach", Ecological Indicators, 89, pp. 84-100 (2018).

32. Alikhani-Kooshkak, R., Tavakkoli-Moghaddam, R., Jamili, A., and Ebrahimnejad, S. "Multi-objective mathematical modeling of an integrated train makeup and routing problem in an Iranian railway company", Scientia Iranica, 26(6), pp. 3765-3779 (2019). DOI: 10.24200/SCI.2018.20782

\section{Biographies}

Ali Mahmoodirad is an Assistant Professor of Applied Mathematics (Operations Research) at MasjedSleiman branch of Islamic Azad University in Iran. His research interests include fuzzy mathematical programming, supply chain management, and decomposition methods. He has published research articles in international journals of mathematics and industrial engineering.

Sadegh Niroomand is an Assistant Professor of Industrial Engineering at Firouzabad Institute of Higher
Education in Iran. He received his $\mathrm{PhD}$ degree in Industrial Engineering from Eastern Mediterranean University, Turkey, in 2013. His research interests are operations research, fuzzy theory, and exact and metaheuristic solution approaches.

Farhad Hosseinzadeh Lotfi is currently a Full Professor in Mathematics at the Science and Research Branch of Islamic Azad University (IAU), Tehran, Iran. He received his BSc degree in Mathematics from Yazd University, Yazd, Iran, in 1992; his MSc in Operations Research from IAU, Lahijan, Iran, in 1996; and PhD in Applied Mathematics (Operations Research) from IAU, Science and Research Branch, Tehran, Iran, in 2000. His major research interests are operations research and data envelopment analysis. He has published more than 300 scientific and technical papers in leading scientific journals, including European Journal of Operational Research, Computers and Industrial Engineering, Journal of the Operational Research Society, Applied Mathematics and Computation, Applied Mathematical Modelling, Mathematical and Computer Modelling, Journal of the Operational Research Society of Japan, etc. He is Editor-in-Chief and a member of the editorial board of the Journal of Data Envelopment Analysis and Decision Science. He is also Directorin-Charge and a member of the editorial board of the International Journal of Industrial Mathematics. Some of his publications are available on the following link: http://scholar.google.com/citations?user=gc qn8gAAAAJ\&hl=en. 